\title{
Sistema de Vigilância Alimentar e Nutricional na implementação do programa Leite é Saúde: avaliação em municípios baianos
}

\author{
Nutritional Surveillance System at the program \\ "Leite é Saúde/milk is health" for underweight children \\ and pregnant women: evaluation at Bahia, Brazil
}

Luciana Alaíde Alves SANTANA 1,2

Sandra Maria Chaves dos SANTOS 1

R E S U M O

\section{Introdução}

Este estudo buscou avaliar como se deu o processo de implantação e execução do Sistema de Vigilância Alimentar e Nutricional em municípios baianos onde o Programa "Leite é Saúde" estava funcionando.

\section{Métodos}

Foram realizadas visitas, observações e entrevistas com profissionais de saúde, no período de 1997-1998. Foram visitados 44 municípios, dos quais 35 possuíam o programa estabelecido. Dentre estes, $43 \%$ realizaram diagnóstico nutricional simultaneamente à implantação na ocasião do programa.

\section{Resultados}

O Sistema de Vigilância Alimentar e Nutricional estava funcionando em 22\% dos municípios, parcialmente implantado em 34\% e em 38\% dos municípios não dispunha deste. Em 6\% dos municípios não foi possível obter informações sobre o Sistema de Vigilância Alimentar e Nutricional. Dos municípios que possuíam o sistema parcialmente implantado, a principal deficiência estava na não utilização das informações para reprogramar atividades.

\section{Conclusão}

O sistema de informações sobre o estado nutricional de crianças reproduziu problemas conhecidos em outros sistemas de informação. A exigência de estabelecer-se o Sistema de Vigilância Alimentar e Nutricional para

\footnotetext{
1 Departamento de Saúde, Universidade Estadual de Feira de Santana. Feira de Santana, BA, Brasil.

2 Núcleo de Nutrição e Políticas Públicas, Departamento da Ciência da Nutrição, Escola de Nutrição, Universidade Federal da Bahia. Av. Araújo Pinho, 32, Canela, 40110-150, Salvador, BA, Brasil.
} 
assinar-se o convênio com o programa "Leite é Saúde", não garantiu a sua real implantação. Com isso, não se produziram mudanças na prática, já que não se utilizou o perfil epidemiológico nutricional local, para atuar sobre problemas de alimentação e nutrição.

Termos de indexação: Sistema de Vigilância Alimentar e Nutricional, política de nutrição, sistemas de informação.

\section{A B S T R A C T}

\section{Introduction}

The purpose of this study was to analyze how the implementation process and carrying out of the Nutritional Surveillance System (or - Information System on children nutrition status) came to pass in municipalities of the State of Bahia, where a supplementary feeding program ("leite é saúde", delivering milk and soy oil) had been established implemented.

\section{Metods}

From 1997 to 1998, a retrospective documental research was performed, including visits, observation and at semi-structured interviews with health professionals. Thirty-five municipalities out of 44 visited had already implemented the program; $43 \%$ of those had performed nutrition diagnosis at the start-up of the feeding and nutrition program. It was not possible to obtain information about Nutritional Surveillance System in $6 \%$ of the municipalities.

\section{Results}

The Nutritional Surveillance Systems had been implemented in 22\% of the towns; $34 \%$ had it only partially implemented and 38\% had not implemented it yet. The main deficiency found in the health service provided in municipalities that had the partially implemented stemmed from the very lack of use of Nutritional Surveillance System's information for planning further actions.

\section{Conclusion}

The Information System on children nutrition status reproduced the problems already known to exist in regard to other information systems. The requirement to implement Nutritional Surveillance System in order to establish a covenant did not accomplish to assure the actual implementation of the Information System. Since the program did not make use of information on epidemiologic nutrition profile in order to detect and solve problems in feeding and nutrition practices, it failed to introduce further practical changes.

Index terms: nutritional surveillance, nutrition policy, information systems.

\section{N T R O D U Ç Ã O}

O agravamento das más condições de vida nos países não desenvolvidos, com o aumento cada vez mais alarmante de desempregados, analfabetos e desnutridos, tem sido pauta de conferências internacionais organizadas por agências como Food and Agriculture Organization (FAO), Organização Mundial da Saúde (OMS) e Fundo das Nações Unidas para Infância (UNICEF).
Três conferências mundiais, que aconteceram nas décadas de 70 e 80, podem ser consideradas como marcos na consolidação do interesse político internacional pela questão da fome e desnutrição. Foram elas: Conferência Mundial de Alimentação (FAO - Roma, 1974), Conferência de Alma-Ata (OMS - União Soviética, 1978) e Revolução pela Sobrevivência e Desenvolvimento da Criança (UNICEF - 1983)'1. 
Em 1974 na Conferência Mundial de Alimentação, em Roma, foi formalizada a proposta de vigilância nutricional (VN), fazendo-se uma transposição do conceito de vigilância das enfermidades, sendo que, nos países subdesenvolvidos a VN ganhou caráter emergencial, devido às precárias condições de vida de grupos vulneráveis. Portanto, a VN foi apresentada nesta ocasião como um sistema de informação (SI), um sistema intersetorial de coleta, processamento e análise de informações que teria como objetivo geral promover informações contínuas sobre o estado nutricional de populações, que serviriam de base para tomada de decisões dos responsáveis pela formulação de políticas de alimentação e nutrição².

No Brasil, em 1972, o Instituto Nacional de Alimentação e Nutrição (INAN) desenvolveu um ante projeto do Sistema de Vigilância Alimentar e Nutricional (SISVAN), mas a primeira tentativa de implantação do sistema não obteve êxito ${ }^{2,3}$.

Posteriormente, experiências de implantação da vigilância alimentar e nutricional aconteceram nos estados da Paraíba e Pernambuco, no período de 1983 e 1984, sob a orientação do Instituto Nacional de Alimentação e Nutrição (INAN). Todavia, quando o fluxo de recursos cessou, as experiências findaram na fase de experimentação.

Ainda na década de 80, com o apoio do Fundo das Nações Unidas (UNICEF), surgiram três novos projetos em Pernambuco, Ceará e São Paulo, ligados às universidades e a grupos de pesquisa. Estas experiências pouco avançaram, em parte, devido a uma precária institucionalização da proposta. Batista-Filho', analisando as experiências daquela década, destacou a insuficiente sustentação política do sistema, sua baixa cobertura geográfica, enfoque centrado na geração de informações antropométricas, desvalorização do processo de interpretação e falta de ações concretas a partir dos dados gerados.

$\mathrm{Na}$ década de 90, destacou-se a institucionalização do SISVAN como responsabilidade formal do Ministério da Saúde. Porém, o sistema só ganhou maior dimensão a partir de 1993, quando foi vinculado ao Programa de Atenção aos Desnutridos e Gestantes em Risco Nutricional - "Leite é Saúde".

Em seu discurso oficial desse período, o INAN reconhecia que as ações de vigilância nutricional eram poucas ou sequer executadas nos municípios. Diante desta constatação, planejou-se a integração da VN ao Programa do "Leite é Saúde", crendo-se que este programa funcionaria como catalisador daquela atividade ${ }^{4}$.

O Programa "Leite é Saúde" teve início em 1993, no governo do presidente Itamar Franco, que em seu discurso de posse atribuiu prioridade ao combate à fome. A existência de uma proposta do Partido dos Trabalhadores (PT) para adoção de uma política de Segurança Alimentar, criou um ambiente propício à instauração de uma nova experiência de participação. Essa participação consubstanciou-se, em primeiro lugar, na elaboração de um conjunto de compromissos de ação e na montagem de um Conselho de Estado - o Conselho Nacional de Segurança Alimentar $(\text { CONSEA })^{5}$. Este conselho teria função de consulta, assessoria e indicação de prioridades ao Presidente da República, representando uma parceria entre ministros de Estado e personalidades notáveis, identificadas com vários setores da sociedade civil e indicadas pela ação da cidadania ${ }^{6}$. A ação do CONSEA gerou o "Plano de Combate à Fome e à Miséria". Como linha de atuação do Ministério da Saúde no plano, foi instituído o Programa de Atendimento aos Desnutridos e às Gestantes em Risco Nutricional - "Leite é Saúde" ${ }^{5}$. Os objetivos básicos do programa eram: reduzir a prevalência de desnutrição; reforçar a prestação de ações básicas de saúde e contribuir para a implementação do Sistema Único de Saúde (SUS), no que se refere à municipalização e reorganização de serviços. O público alvo eram crianças abaixo do percentil 10 (indicador P/I) na faixa etária de 6 a 23 meses. Estes deveriam receber, diariamente, $120 \mathrm{~g}$ de leite em pó ou 1 litro de leite fluido pasteurizado e uma lata de 
óleo/mês; um irmão da criança, com baixo peso e na faixa etária de 2 a 5 anos, assim como gestantes em risco nutricional, deveriam receber metade da dosagem de leite/dia referida acima sem receber óleo ${ }^{4}$.

O município, para se credenciar a receber os recursos destinados à execução do Programa "Leite é Saúde", deveria, previamente, realizar um diagnóstico antropométrico da população materno-infantil e gerar informações mensais do estado nutricional de crianças e gestantes atendidas em Unidades Básicas de Saúde (UBS), com vistas a ter um sistema que informasse continuamente sobre o perfil antropométrico de gestantes e de crianças menores de cinco anos.

Foi elaborado pelo INAN um 'Roteiro para Implantação da Vigilância Nutricional para o Programa "Leite é Saúde"'. Neste documento, além da indicação dos responsáveis técnicos pela Vigilância Nutricional nos municípios, estes eram instruídos sobre a necessidade da pesagem e da análise da adequação do peso das crianças do município; como convocar a clientela que não freqüentava as Unidade Básica de Saúde (UBS); quais os locais onde poderia acontecer a pesagem das crianças e quais os itens que deveriam constar no plano para o levantamento a ser realizado (quando e onde, quem realizou, como foram chamadas as criançasigestantes para pesagem, que tipo de balança se utilizou). Anexados ao documento, seguiam os mapas mensais do SISVAN, instrumentos que deveriam ser utilizados para anotar o estado nutricional de crianças/gestantes atendidas nas UBS do município7.

Nestes moldes, o SISVAN ganhou duas dimensões; uma mais ampliada, que buscava obter um perfil antropométrico de crianças e gestantes de uma determinada localidade ou micro-localidades. E outra, que visava obter informações sobre o estado nutricional do público-alvo, a fim de constituir bancos de dados, cuja informação seria restrita aos usuários dos serviços de saúde. Estes, utilizariam tal informação, como instrumento gerencial para planejamento e programação local.
Portanto, este sistema, operando em uma situação real e tendo subjacente o conceito de vigilância epidemiológica, teria como elementos básicos a geração, o processamento e a análise de informações que serviriam de subsídios para reorganização das ações específicas necessárias à atenção integral à saúde das crianças e do pré-natal. Mais amplamente, tais informações seriam importantes para instrumentalizar a tomada de decisões no momento da formulação de políticas de alimentação e nutrição no município, no estado e no país.

Nas primeiras avaliações do Programa do "Leite é Saúde", realizadas por Peliano ${ }^{8}$ e Taddei et al. ${ }^{9}$, a integração do SISVAN ao Programa não foi considerada como positiva. Estes autores observaram que o Programa pouco contribuiu para a implantação do sistema nos municípios.

Em outra análise do processo de implantação do SISVAN integrado ao programa "Leite é Saúde", realizada pela coordenação do mesmo no Ministério da Saúde, os autores concluíram que a aliança possibilitou a difusão da proposta de VN no país. Contudo, ressaltaram que a associação com o Programa, exigindo a implantação do SISVAN como condição para assinatura do convênio, banalizou a proposta que passou a ser executada como uma exigência, sem objetivos práticos, e não como um elemento importante para a definição de políticas públicas na área de alimentação e nutrição no país ${ }^{3}$.

As avaliações feitas do SISVAN despertaram a necessidade de maiores investigações sobre como se deu a sua integração com o Programa "Leite é Saúde" nos espaços municipais. Buscava-se uma melhor caracterização do seu processo de implantação, considerando-se ainda a forma como os dados eram obtidos e qual o uso destas informações nos municípios. Portanto, no presente trabalho, pretendeu-se analisar o processo de implantação e execução do SISVAN em um conjunto de municípios baianos, membros do Programa Comunidade Solidária (PCS), onde o Programa "Leite é Saúde" estava sendo executado. 


\section{CASUÍSTICAE MÉTODOS}

Para esta pesquisa, foram visitados 44 municípios, escolhidos aleatoriamente dentre os 96 contemplados pelo PCS na Bahia, no período de 1997-1998.

O procedimento para a coleta de dados foi o registro das informações sobre a existência de um plano de implantação do SISVAN e de banco de dados sobre o estado nutricional de crianças e gestantes, realizado pelo pesquisador em formulário específico, nas UBS. Sobretudo, buscou-se registrar a existência de um sistema de informações sobre o estado nutricional, manual ou informatizado, que pudesse servir de base à implementação do Programa "Leite é saúde" e à reorganização dos cuidados com a saúde da criança e do pré-natal. Além deste levantamento de informações secundárias, foram realizadas entrevistas com secretários de saúde e técnicos envolvidos na atenção básica à saúde da criança em Unidades Básicas de Saúde, de forma a complementar a análise.

Esta análise foi realizada em duas dimensões, uma que considerou a implantação do sistema de base populacional e uma outra dimensão que analisou a sua implantação como instrumento gerencial nas UBS. Para observar as condições de implementação do SISVAN de base populacional, primeiramente, investigou-se se existia um plano de implantação do SISVAN e se o diagnóstico antropométrico de crianças menores de cinco anos e gestantes no município, se realizava anteriormente à implantação do Programa "Leite é Saúde". Quanto à implantação do SISVAN como instrumento gerencial nas UBS, foi necessário verificar se existiam certas condições, as quais, se presentes na organização do Sistema de Vigilância Alimentar e Nutricional da UBS em questão, atenderiam aos objetivos formais propostos para o sistema. Tendo como referência o conceito de VN, tais objetivos incluiriam a utilização das informações obtidas pelo SISVAN como instrumento gerencial das UBS. As condições a serem observadas, referiam-se à coleta de informações sobre estado nutricional (coleta de dados realizada nas unidades de saúde, no domicílio, ou na comunidade), ao processamento dos dados (utilização de instrumento oficial de registro - Ministério da Saúde), à análise (discussão das informações por profissionais das UBS) e à utilização da informação na UBS (transformá-las em instrumentos para a tomada de decisões).

A classificação da situação de implantação foi feita obedecendo ao seguinte critério: caso a UBS do município apresentasse todas as condições descritas acima, considerava-se o SISVAN implantado; se uma ou mais condições estivessem ausentes, considerava-se o SISVAN parcialmente implantado; se não apresentasse nenhuma das condições, não implantado.

\section{RES U LT A D O S}

Foram visitados 44 municípios de diferentes regiões do estado da Bahia, dos quais 35 possuíam o Programa "Leite é Saúde" implantado na época da visita; em 6 deles o programa não estava implantado na ocasião da visita; em outros 3 não foi possível obter informações sobre a implantação do programa.

Para a análise da situação de implantação do SISVAN, teve como recorte principal na delimitação das áreas a serem pesquisadas, a existência no local do programa "Leite é Saúde". Como aquele SI deveria estar vinculado ao programa, não foram considerados os municípios onde este não existia; foram considerados apenas os 35 municípios onde funcionava o "Leite é Saúde".

Para uma análise das condições de implantação do SISVAN de base populacional, ou seja, como sistema de informações sobre o do estado nutricional de crianças, elaborado a partir de diagnóstico antropométrico realizado em comunidades ou em todo o município, foram considerados os 35 municípios citados.

Primeiro observou-se se existia um plano municipal de implantação do SISVAN, no qual 
seriam descritos os procedimentos para realização do diagnóstico antropométrico da população infantil (menores de cinco anos) e de gestantes. Somente em 43\% dos 35 municípios com o programa "Leite é saúde" implantado, os informantes tinham conhecimento da existência de um plano de implantação do SISVAN e da realização do diagnóstico nutricional.

Em 20\% dos municípios estudados, o programa foi implantado sem que o requisito de implantação do SISVAN fosse atendido (Tabela 1). Esta constatação do não cumprimento daquela exigência ao ser instituído o programa "Leite é Saúde", confirma os resultados relatados por Tadde $^{9}$, na avaliação do programa, realizada por ele em 16 municípios brasileiros.

Em 37\% dos municípios, os entrevistados não souberam informar sobre a existência do plano ou sobre a realização do diagnóstico antropométrico, alegando que o programa de recuperação nutricional em questão tinha sido implantado na gestão anterior e o atual gestor e os técnicos envolvidos na execução do mesmo nas UBS, não tinham qualquer informação sobre como se deu o processo de implantação do programa "Leite é Saúde" ou do SISVAN (Tabela 1).

Pode-se observar, por estas informações, que os indivíduos diretamente envolvidos na gestão e execução do programa "Leite é Saúde" não tinham conhecimento da realização de um diagnóstico nutricional prévio à implantação do programa; isto, tanto pode indicar que tal diagnóstico não aconteceu e por isso não havia registros, como pode indicar que os atuais envolvidos nas ações de recuperação nutricional no município, pouco valorizam a informação como instrumento fundamental para planejamento e programação das ações de saúde.

Para análise do SISVAN como instrumento gerencial nas unidades básicas de saúde, foram considerados 32 municípios, uma vez que, das 35 localidades onde o programa "Leite é Saúde" estava sendo executado, três, não apresentaram integração entre o programa e as UBS. Em $6 \%$ destes 32 municípios não foi possível obter informações sobre o SISVAN; em $22 \%$ o SISVAN estava implantado; em 34\%; parcialmente implantado; não estava implantado em $38 \%$ dos municípios, considerando-se as condições descritas na metodologia.

O percentual de municípios em que o SISVAN não foi implantado nas UBS, foi superior a todas as outras classificações; considerando-se as condições definidas neste estudo, isto significa que em grande parte (38\%) dos municípios, nem o registro de informações nem o envio das mesmas para os níveis superiores estavam sendo realizados.

Quanto aos municípios classificados como tendo o SISVAN parcialmente implantado, as condições de implantação menos observadas foram a análise e a utilização das informações nas UBS para programação de ações. Pode-se atribuir estes resultados à pequena apropriação por parte dos profissionais de saúde de metodologias de planejamento de base local ${ }^{10}$; já que o modelo assistencial de saúde predominante não valorizava linhas de atuação que utilizassem a informação como suporte gerencial do nível local, suporte importante no processo de planejamento, acompanhamento e tomada de decisões.

Tabela 1. Situação dos Municípios com Programa do Leite implantado segundo a elaboração do plano de implantação do SISVAN. Salvador, 2000.

\begin{tabular}{|c|c|c|}
\hline Elaboração Plano Implantação do SISVAN & $\mathrm{n}$ & $\%$ \\
\hline Municípios que elaboraram plano de implantação do SISVAN & 15 & 43 \\
\hline Municípios que não elaboraram plano de implantação do SISVAN & 7 & 20 \\
\hline Municípios que não souberam informar sobre a elaboração de um plano de implantação do SISVAN & 13 & 37 \\
\hline Total & 35 & 100 \\
\hline
\end{tabular}


Merece destaque, como componente causal do incipiente uso da informação como ferramenta para formulação de políticas públicas locais, a tradicional centralização dos processos de formulação e gerenciamento das políticas sociais no país, que deixa para o município apenas a função de executar algo pronto, concebido fora da sua realidade e imposto de cima para baixo. Pretendeu-se reverter esta situação a partir da Constituição de 1988, com um rearranjo do pacto federativo brasileiro no sentido de desenvolver um processo descentralizador; no entanto, tal processo, ao longo destes anos tem enfrentado dificuldades em todas as esferas de governo. $\mathrm{Na}$ realidade de cada município pode-se destacar como entrave o insuficiente planejamento para a descentralização, em parte, devido ao caráter vertical dos programas de nutrição e/ou por características próprias do arranjo institucional ${ }^{5}$.

\section{O N CLUS Ã O}

Os resultados obtidos apontaram para uma incipiente implantação do SISVAN nos municípios visitados, seja na sua forma de diagnóstico da população materno-infantil, cumprindo o pré-requisito para implantação do Programa "Leite é Saúde", seja como instrumento gerencial nas unidades básicas de saúde.

Observou-se ainda que a exigência de implantação do SISVAN para assinatura do convênio do Programa "Leite é Saúde" não garantiu a sua real implantação, uma vez que $20 \%$ dos municípios não realizaram diagnóstico antropométrico anterior à implantação do programa de recuperação nutricional, comprometendo portanto o papel do SISVAN como instrumento de ação para definição de políticas e revelador de tendências na área de alimentação e nutrição.

Em 38\% dos municípios as informações não estavam sendo geradas nas UBS onde o referido programa estava funcionando. Observou-se que o SISVAN obteve pequena institucionalização, bem como, no geral, limitou-se á geração de informações, na medida em que foi pouco utilizado como indicador de nós críticos na operacionalização de ações específicas de atenção integral à criança.

Verificou-se na análise do sistema como instrumento gerencial, que houve uma reprodução de problemas conhecidos em outros sistemas de informação. Estes tais problemas, destacam-se a produção de informações no nível local, muitas vezes de qualidade discutível, e o seu encaminhamento para uma instância superior sem que estes dados tenham sido manuseados e transformados em ações concretas pela equipe de saúde do município.

As informações obtidas quanto à utilização dos dados gerados confirmam que o SISVAN ainda não conseguiu superar problemas que apresentou desde sua origem no Brasil, como já foi demonstrado no estudo de Batista-Filho ${ }^{1}$ das primeiras experiências de implantação do sistema. Em sua análise, o autor enfatiza que a análise e interpretação dos dados obtidos tem ficado em segundo plano e tais dados, portanto, não produzem ações concretas.

Observou-se, então, que a exigência formal de implantação do SISVAN não foi suficiente para garantir a geração e utilização local de informações sobre o estado nutricional de crianças/gestantes nos municípios. Deve-se considerar que o SISVAN se inseriu em realidades que não utilizavam a informação como um instrumento gerencial e que, a incorporação desta prática demanda mudanças que não dependem somente de normas.

A vinculação do SISVAN com o programa "Leite é Saúde" também pode ser apreciada a partir da lógica de cada uma das ações: enquanto o programa teve o desenho de uma ação setorial, paliativa e com foco na recuperação, o SISVAN parte da lógica da vigilância epidemiológica, que indica a organização necessária das ações na área de alimentação e nutrição com base no perfil epidemiológico nutricional.

Esta diferenciação seria fundamental e instrumental para quem está na ponta opera- 
cionalizando as ações; ou seja, o entendimento desta distinção conceitual das duas ações, por parte dos profissionais de saúde, poderia contribuir para garantir a implantação do SISVAN, de forma mais eficaz que a exigência formal de integração deste a um programa de suplementação alimentar.

Cabe concluir ressaltando que, além dos avanços alcançados na última década, é necessário evoluir transformando o SISVAN em um sistema útil e capaz de produzir mudanças na prática em nutrição, por meio da utilização do perfil epidemiológico nutricional da população infantil, visando atuar sobre problemas de alimentação e nutrição dos municípios ou micro localidades.

\section{A GRADECIMENTOS}

À Agencia FINEP por ter financiado o Projeto de Avaliação de Políticas Públicas no Estado da Bahia, do qual este estudo faz parte.

\section{REFER Ê N CIAS}

1. Batista Filho $M$, et al. Vigilância alimentar e nutricional: antecedentes, objetivos e modalidade. A VAN no Brasil. Cad Saude Publica 1993; 9(1) 99-105.

2. Castro IRR. Vigilância Alimentar e Nutricional: limitações e interfaces com a rede de saúde. Rio de Janeiro: Fiocruz; 1995.

3. Oliveira e Silva $D$, et al. O Processo de Implantação do SISVAN no Brasil e o Programa do Leite é Saúde. Bol Nac SISVAN 1996; 1(2).
4. Ministério da Saúde. Programa de atendimento aos desnutridos e gestantes em risco nutricional: roteiro para implantação da vigilância nutricional. Brasília; 1993.

5. Santana LAA. O Programa de atenção aos desnutridos e gestantes em risco nutricional "Leite é saúde"- em Salvador Bahia, 1994-99: Limites e perspectivas da descentralização em políticas de saúde [dissertação]. Salvador: Universidade Federal da Bahia, Instituto de Saúde Coletiva; 2000.

6. Instituto de Pesquisa Econômica Aplicada. Um balanço das ações do governo no combate a fome e a miséria. Brasília; 1994.

7. Ministério da Saúde. Programa de atendimento aos desnutridos e gestantes em risco nutricional. Brasília; 1993.

8. Peliano AM. O mapa da fome; subsídios à formulação e uma política de segurança alimentar: documentos de política, n.14. Brasília: IPEA; 1993.

9. Taddei JAA, et al. Avaliação operacional do programa de atendimento aos desnutridos e às gestantes em risco nutricional - leite é saúde. São Paulo: Unifesp; 1996.

10. Teixeira CF. Planejamento e programação situacional em distritos sanitários: metodologia e organização. In: Mendes EV. Distrito Sanitário: o processo social de mudança das práticas sanitárias do Sistema Único de Saúde. São Paulo: Hucitec; 1995.

Recebido para publicação em 17 de setembro de 2002 e aceito em 7 de agosto de 2003. 